\title{
Drug-Induced Leukoencephalopathy: MR Follow-Up After Methotrexate Therapy
}

\author{
Teresa Popolizio, Nicola Sforza, and Anna Maria Pennelli
}

- 52-year-old patient with Non-Hodgkin lymphoma.

- MR follow-up in chronic phase after treatment with methotrexate.

\section{Late Follow-Up After Chemotherapy}
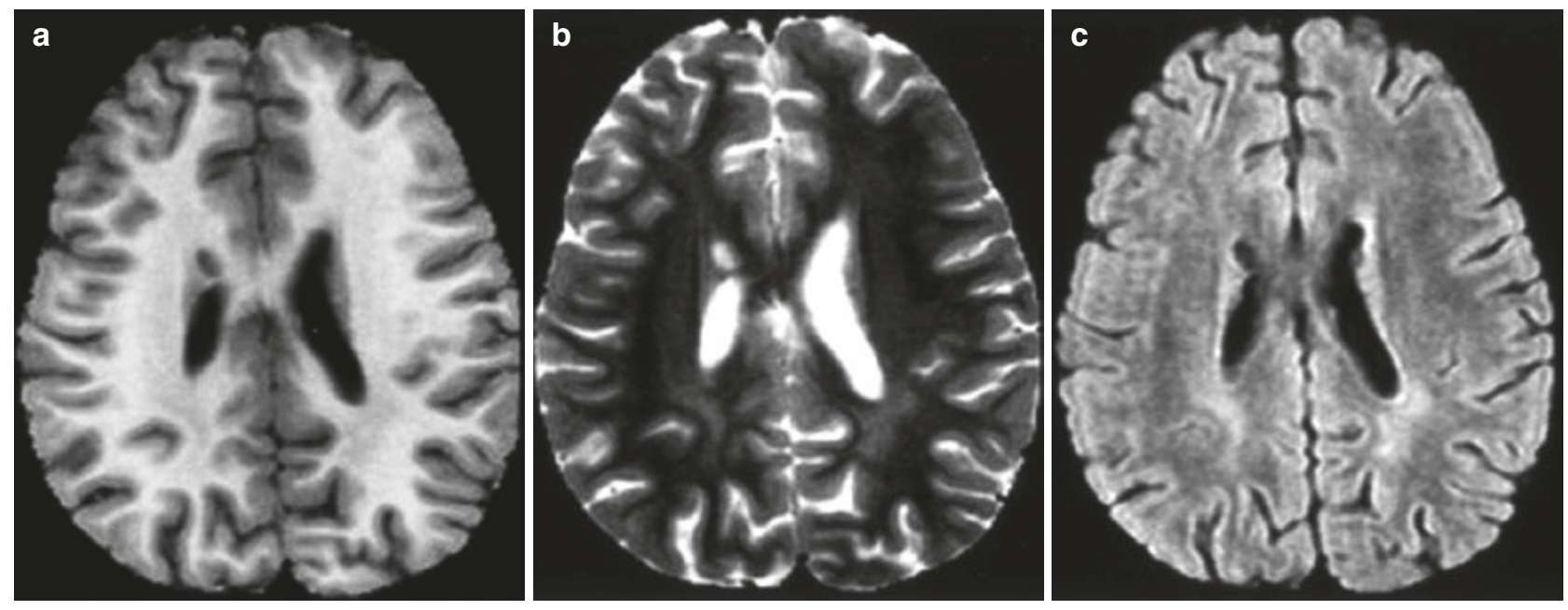

Fig. 51.1 MR SE T1-weighted (a), FSE T2-weighted (b), and FLAIR (c) sequences. Slight and circumscribed signal alteration (hypointensity in $\mathrm{T} 1$ and T2, hyperintensity in FLAIR) can be appreciated in the posterior periventricular white matter bilaterally

T. Popolizio $(\varangle) \cdot$ N. Sforza

Department of Neuroradiology, Fondazione IRCCS “Casa Sollievo della Sofferenza”, San Giovanni Rotondo, FG, Italy
A. M. Pennelli
Department of Radiology, Fondazione IRCCS "Casa Sollievo della
Sofferenza”, San Giovanni Rotondo, FG, Italy 